\title{
e-Phaïstos
}

e-Phaïstos Revue d'histoire des techniques / Journal of the history of technology

IV-2 | 2015

Patrimoines de l'eau

\section{Des modes d'existence du geste technique}

On the Modes of Existence of Technical Gesture

\section{Anne-Françoise Garçon}

\section{CpenEdition \\ Journals}

Édition électronique

URL : http://journals.openedition.org/ephaistos/797

DOI : 10.4000/ephaistos.797

ISSN : 2552-0741

Éditeur

IHMC - Institut d'histoire moderne et contemporaine (UMR 8066)

Édition imprimée

Date de publication : 1 octobre 2015

Pagination : 84-92

ISSN : 2262-7340

Référence électronique

Anne-Françoise Garçon, «Des modes d'existence du geste technique », e-Phaïstos [En ligne], IV-2 I

2015, mis en ligne le 29 novembre 2016, consulté le 11 novembre 2019. URL : http://

journals.openedition.org/ephaistos/797; DOI : 10.4000/ephaistos.797 


\title{
Des modes d'existence du geste technique
}

\author{
Anne-Françoise Garçon \\ Équipe d'Histoire des Techniques / IHMC \\ CNRS-Université Paris 1 Panthéon- \\ Sorbonne
}

En 2005, il y a dix ans donc, paraissait Stone Knapping: The Necessary Conditions for a Uniquely Hominid Behaviour, ouvrage collectif dirigé par Valentine Roux et Blandine Bril ${ }^{1}$. Stone Kapping, le débitage des pierres, c'est le geste technique des débuts de l'histoire humaine, celui de la charnière entre les primates supérieurs, humains et nonhumains ${ }^{2}$. En quoi, interrogeait l'ouvrage, le geste technique des sociétés humaines se distingue-t-il de celui des sociétés non-humaines, aux deux plans de la phylogenèse (qui est l'histoire de la constitution de l'espèce) et de l'ontogenèse (qui est l'histoire de la constitution de l'individu dans l'espèce) ? Autrement dit : qu'est-ce qu'un geste technique humain ? Comment l'humain le conçoit-il ? De quelle manière est-il facteur d'individuation?

L'ouvrage renouvelait la compréhension de la relation entre geste technique et cognition. Il mettait à jour en effet la qualité d'agrégat du geste technique qui met en jeu simultanément les contraintes et les potentialités matérielles, la capacité motrice et musculaire des divers hominidés et leur capacité neurocognitive à interpréter, à anticiper et à adapter. Le débitage des silex est un savoir-faire sophistiqué qui requiert une interprétation du matériau et un ajustement corrélatif des mouvements, un savoir-faire qui implique une procédure complexe et durable d'apprentissage. Il démontrait enfin que cet apprentissage porte sur la globalité de l'objectif recherché, sur le but de l'action et non sur le geste lui-même. Ce geste que d'aucuns qualifieront d'élémentaire, mobilise et formate d'emblée les capacités matérielles, motrices et neurocognitives

des hominidés qui l'apprennent et l'exécutent et qui enseignent comment l'exécuter.

L'étude confortait les analyses de François Sigaut, Jean-Pierre Warnier et Michel Simondon (Michel et non Gilbert, son père). Un marteau ne sert à frapper que s'il sert... en frappant, observe François Sigaut dans un article devenu célèbre3. L'apprenti n'apprend pas à se servir d'un marteau, ou d'un percuteur, en soi ; il apprend se servir d'un marteau ou d'un percuteur pour... planter un clou, débiter un silex, tailler un biface, forger un briquet, etc. Le geste inventé, appris ou redécouvert, incorpore au sens propre du terme l'expérience de la matière, de l'outil avec l'intentionnalité qui l'anime.

« La matière médiatise l'hominisation et la subjectivation, elle ne fait pas double emploi avec le discours et constitue un espace autonome de production du sens corporel pré-linguistique par la différenciation/structuration intentionnelle du cadre matériel, la mise en objets et l'action. » 4

Et le geste, pour être technique, incorpore durablement cette topique matière/action/pensée intentionnelle.

«La technique, explique Michel Simondon, se définit essentiellement par ses schèmes de fonctionnement, ses normes opératoires internes. Il y a normes et normativités techniques dans la mesure où le sens de l'action humaine transformatrice s'oriente selon certaines solutions qui la réverbèrent et qui ne sont pas neutres. La normativité technique n'est ni industrielle seulement, ni économique. » 5

Ces avancées majeures dans la compréhension du geste technique sont le produit d'une méthode 
exemplaire que je résumerai ainsi : fixation d'un socle rigoureux d'analyse du geste technique ; mise en jeu de ce socle dans des études pluridisciplinaires les unes croisant anthropologie, neurocognition, éthologie, et études préhistoriques, les autres croisant anthropologie, neuro-cognition, psychologie de l'enfant et ergonomie ${ }^{6}$. L'historien des techniques, en regard, se heurte à plusieurs difficultés quand il cherche à faire du geste technique un objet d'histoire. Première d'entre elles, la durée. Étudier d'un point de vue historique les gestes, les outils et les procédés qui composent et définissent les pratiques des métiers oblige à composer avec la très longue durée. L'historien de l'agir opératoire risque fortement de fausser ses interprétations s'il enferme sa recherche dans les périodes institutionnelles. Et il se prive d'éléments déterminants de comparaison. Il lui faut donc s'éloigner de la périodisation qui, en France tout particulièrement, structure la science historique dans son dispositif institutionnel7.

La question des sources complique le jeu. Les sources écrites qui fondent la pratique historienne, font souvent défaut pour l'étude des techniques, soit par leur absence, soit parce qu'elles sont peu explicites. Elles manquent à fournir les indications qui aideraient à concevoir les gestes pratiqués dans des cultures techniques différentes de la nôtre ${ }^{8}$. Et, aucun texte technique ne procure à l'historien la dimension sensible, regard, odeur, écoute des bruits, qui accompagne et conduit le geste et l'outil. L'analyse historique du geste technique ne peut se faire sans une relation directe, sensorielle, à la pratique. La reconstitution visuelle et sonore d'un quartier parisien, orchestrée par Mylène Pardoen dans le cadre du projet Bretez, est pilote en la matière 9 . Et depuis une dizaine d'années, les archéologues, les historiens et les restaurateurs utilisent la CAO pour affiner leur connaissance du geste technique, que ce soit dans les mines romaines de Rosia Montana en Roumanie ${ }^{10}$, dans l'atelier gallo-romain de fonte des grands bronzes du Grand Hôtel de Bordeaux ${ }^{11}$ ou dans les ateliers des Usines Renault au début du $\mathrm{XX}^{\mathrm{e}}$ siècle ${ }^{12}$. La restitution en $3 \mathrm{D}$ de la grotte Chauvet-Pont d'Arc en Ardèche a permis à Gilles Tosello, plasticien et chercheur associé au CNRS, de comprendre le geste des grands artistes du paléolithique ${ }^{13}$. Par ce biais, tous ont pu vérifier, infirmer et corriger des hypothèses et améliorer l'intelligence de la relation geste/outil/pensée opératoire dans les environnements de travail disparus.

Le coût de ces dispositifs limite leur emploi toutefois. La planche de salut, dans le quotidien de la recherche historique, c'est l'iconographie. Qui, travaillant sur la poterie, la briqueterie, la fonte des grands bronzes, les métiers du métal, etc. n'a pas eu recours aux multiples planches techniques éditées entre $\mathrm{XVI}^{\mathrm{e}}$ et $\mathrm{XVIII}^{\mathrm{e}}$ siècle, De re metallica de G. Agricola, Descriptions des Arts \& Métiers de l'Académie des Sciences, Encyclopédie de Diderot et d'Alembert, Encyclopédie Méthodique éditée par Panckoucke ? J'ai souvenir, lorsque dans les années 1990 j'étudiais les techniques de production des métaux non ferreux à l'époque moderne, de cette habitude que nous avions prise dans les fouilles minières et paléométallurgiques d'épingler au mur de la salle commune les planches du De re metalli$c a$. De la sorte, chacun pouvait s'imprégner de la représentation des ateliers et réfléchir sur les espaces techniques. Cette habitude, je l'ai gardée dans mon bureau, jusqu'au jour où, enfin, j'ai compris que le cœur des techniques de fonte, la maîtrise du procédé résidait dans la manière de donner les vents entre la sole et lit de fusion. Or ce moment crucial de la chaîne opératoire est totalement absent de l'ouvrage, textes et planches comprises ${ }^{14}$ ! G. Agricola n'a jugé utile ni d'en parler, ni de les représenter 15 .

C'est que l'intentionnalité des sources diffère de celle du geste. Il en va de même de l'historicité. Le chercheur se doit de composer avec ces décalages. Décalage dans l'intentionnalité : personne n'a jamais appris à escrimer, à danser, à produire du métal, à faire du judo, du vélo ou du ping-pong en lisant un livre ou une encyclopédie, aussi illustrée soit-elle. Et nous savons tous qu'une recette si 
bonne soit-elle ne fait pas le cuisinier. Pourquoi donc alors coucher sur le papier le geste de l'escrimeur au XVI ${ }^{\mathrm{e}}$ siècle ${ }^{16}$, celui du judoka ou du pongiste dans les années $1960^{17}$ ? Décalage dans l'historicité: le geste technique ne s'inscrit pas d'abord dans une " période » historique; il s'inscrit d'abord dans un régime de pensée et d'agir; et ce régime opératoire, cette " technicité en acte » pour reprendre l'expression de Michel Simondon ${ }^{18}$, le spécifie. En regard, la mise en scène textuelle et/ou iconographique d'un geste technique, comme celle d'ailleurs d'un outil ou d'un procédé s'origine dans une intentionnalité distincte, fruit d'un contexte donné. Et son régime opératoire est nécessairement distinct. Voilà pourquoi l'étude historique doit : 1) Analyser de la manière la plus concrète possible le geste, l'outil ou le procédé considéré de manière à percevoir les possibles et les limites posées par la matérialité ${ }^{19}$ et le traitement qu'en fait la culture qui l'englobe ${ }^{20}$; 2) Apprécier l'intentionnalité, les modalités et les formes de sa description textuelle et/ou iconographique ; 3) Déterminer le régime opératoire qui préside à cette intellectualité scripturaire, par essence distincte de l'intellectualité praticienne ; 4) En évitant de réduire la distinction entre ces deux intellectualités à une simple opposition théorie/pratique. Car écrire n'est pas théoriser en soi ; et pratiquer suppose une théorie implicite.

Il faut comprendre que les modes d'existence du geste technique diffèrent selon les régimes opératoires dans lesquels ils sont intégrés, dans (voire pour) lesquels ils sont exécutés. Les gestes des verriers ou des marbriers, les gestes du charpentier de marine, ceux du chirurgien, les gestes du restaurateur d'art, ceux du maçon, ceux du forgeron, mes propres gestes lorsque je fais du vélo, lorsque je jardine, quand j'écris, quand je conduis ma voiture ou que je fais de la voile, relèvent strictement de la pratique $^{21}$. Leur existence tient à la capacité de chaque opérateur à les produire, à les reproduire, voire à les améliorer et à la capacité du collectif humain concerné à définir une forme de normativité, idéelle et matérielle, qui permettent leur appren- tissage et leur transmission dans la durée, ce qu'est le régime de la pratique. Avec pour but, l'apprentissage et la transmission dans un plan de référence strictement oral, quoique chacun des gestes appris et transmis relève à la fois de l'intelligible et du sensible. Il y a donc moins geste que milieu gestuel, pour suivre Blandine Bril, un milieu gestuel capable de former et d'informer l'opérateur en cristallisant les mots, les sons, les images autour de l'acte et de son intention. C'est le squelette de ce milieu gestuel que l'archéologue retrouve avec une émotion toujours renouvelée lorsqu'il étudie les traces d'outils dans les mines et les carrières anciennes, les traces de scie sur les vieilles charpentes et les graffitis techniques sur les murs en plâtre du Moyen-Age; ou encore l'archéomètre, lorsqu'il repère dans une pointerolle, dans une barre de fer médiévale, les moments de sa forge et parfois les ratés et repentirs.

C'est un autre mode d'existence du geste technique qu'inventent en Europe les lettrés du Xvi ${ }^{\mathrm{e}}$ siècle lorsqu'ils décident de "réduire en art " l'escrime, la danse ou la serrurerie, et de « réduire en science » les pratiques minières et métallurgiques, ou les savoir-faire agricoles ${ }^{22}$. Certains métiers y échappent : personne n'a réduit en art la pratique des métiers féminins, du moins avant l'ère industrielle. Certaines phases des chaînes opératoires y échappent aussi : aucune planche du De re metallica ne donne à voir la finition de la sole de coupellation, autre moment-clé du savoir-faire métallurgique. C'est qu'il appartenait au maîtrecoupelleur de terminer seul le damage de la sole, à l'abri des regards, y compris celui de ses aides ${ }^{23}$. D'autres au contraire sont mis en avant, pour faire comprendre, par exemple, la facilité d'emploi des machines que ces gestes mettent en œuvre, et l'importance du travail qu'elles accomplissent en regard ${ }^{24}$.

La visée de ces écrits n'est pas encyclopédiste. Le but des traités techniques du XVI ${ }^{\mathrm{e}}$ siècle n'est pas de tout dire en laissant au lecteur la liberté d'aller et venir dans le texte au gré de ses envies ou de ses 
besoins, qui est le propre du projet encyclopédique ; il est de rassembler, de constituer une somme donc, en l'ordonnant de manière à ce qu'elle fasse sens. Transcrit, le geste de l'escrimeur devient géométrique. Et cette géométrie confère à l'escrime un sens et une noblesse que la vulgarisation de la pratique et l'interdiction du duel, lui faisaient perdre 25 . Le De re metallica n'est pas une encyclopédie des savoirs miniers mais la première lecture globale de la pratique minéro-métallurgique ordonnée de la mine au métal. Son but clairement affiché : donner ses lettres de noblesses au savoir minérométallurgique, ôter ce caractère sulfureux que les pratiques spéculatives et celle des alchimistes donnaient à la mine et à la métallurgie, procurer durablement des outils de gestion aux entrepreneurs, ingénieurs et administrateurs. L'imprimé technique au XVIe siècle n'est pas utilisé pour former l'opérateur. Il est utilisé pour informer le lecteur, pour l'aviser. Il donne à voir la pratique en même temps qu'il en donne une lecture raisonnée. Ce faisant, il l'enchâsse dans un environnement intellectuel qui échappe au praticien ${ }^{26}$ et lui confère une efficacité d'un nouveau genre, pensée, appréhendée, conçue dans une stricte relation à la technique hors de toute considération religieuse, cosmologique et même socio-professionnelle.

Cette normativité démonstrative et gestionnaire qui caractérise le régime de la technique dans l'Europe de la Renaissance va progressivement évoluer. Ce qui était pour les philosophes, ingénieurs et savants des XVI et XVII ${ }^{\mathrm{e}}$ siècles un outil de gestion des savoirs d'action, devint pour les savants, ingénieurs et administrateurs du XVIII siècle, un outil de gestion de l'action par les savoirs. Dans le dernier $\mathrm{XVII}^{\mathrm{e}}$ siècle, Colbert posait concrètement la question de l'amélioration de la productivité technique par l'organisation rationnelle des pratiques dans les arsenaux 27. La jeune Académie des Sciences se vit confier entre autres missions, la description des Arts \& Métiers ${ }^{28}$. La division des tâches n'était pas en soi une nouveauté. Mais ce que le XVIII' siècle invente, c'est l'inscription de la rationa- lité technique dans une science économique en cours de constitution, à partir de cette culture technique que les ingénieurs et les savants élaboraient depuis un siècle. Jean-Louis Peaucelle a montré l'influence profonde exercée par Jean-Rodolphe Perronet sur Adam Smith29. Pour tout dire, l'inventeur de la fabrique d'épingle comme archétype de l'atelier industriel, c'est le fondateur de l'École des Ponts \& Chaussées. Le régime de la technique, les métiers d'ingénieur et de savant, et l'économie en tant que science de la production, ont entre eux des liens profonds ${ }^{30}$. Bien évidemment, personne au XVIII ${ }^{e}$ siècle ne pouvait imaginer que la machine deviendrait le prescripteur gestuel de l'atelier industriel, et avec elle, l'ingénieur d'industrie, qui porta à son apogée le régime de la technique dans le cadre de l'OST, l'organisation scientifique du travail.

Le chemin de la fabrique d'épingle passe bien sûr par l'Encyclopédie de Diderot et d'Alembert, avec l'article « Épingle » de Delaire, l'article « Épinglier » de Perronet et les planches de Jean-Jacques Goussier. Mais c'est une voie complémentaire que défend Diderot dans deux articles célèbres, les articles «Arts » (terme que l'on utilisait alors pour signifier «technique ») et "Bas ». Pour répondre aux critiques que les deux premiers articles avaient suscitées, il reprend son argumentaire dans l'article «Encyclopédie »31. Fils et petit-fils de coutelier, Diderot connaît la rigueur et la rationalité du travail d'artisan mieux que quiconque parmi les philosophes et savants avec qui il travaille. Et s'il feint d'ignorer l'habitude prise par les philosophes de travailler avec les artisans (Leibniz rappelons-le avait d'abord exercé en tant qu'ingénieur ${ }^{32}$, Francis Bacon pratiquait assidûment l'expérimentation technique, Descartes s'essaya à l'échange avec les artisans, Newton conçut un télescope et Huygens mit au point la première montre à spiral plat avec l'horloger parisien Thuret), c'est parce qu'il propose un autre programme de recherche. La leçon ne vient pas de lui, pour tout dire, mais de Christian Wolff, le grand philosophe allemand, dont il recopie 
l'argumentation quasiment mot pour mot dans l'article «Arts »33. Elle tient en une proposition : il n'y a pas d'un côté la science qui serait théorique et de l'autre les Arts qui seraient pratiques ; il y a deux domaines, le scientifique et le technique, qui possèdent chacun leur théorie et leur pratique. Voilà pourquoi Diderot propose aux philosophes de retourner dans les ateliers. Non pour mettre au point des instruments, organiser des expérimentations ou décrire des procédés 34 , mais pour analyser la pratique des artisans, en extraire les schèmes, les principes d'action, en faire la théorie et les rendre adaptables à l'ensemble des métiers.

Son projet est technologique, dans le sens que Christian Wolff donnait à ce terme d'une science de la pratique, et tout particulièrement de la pratique des métiers. Avec pour visée affirmée, la technique elle-même, et non l'administration des métiers et de leurs savoirs au sens où le développèrent ensuite Johann Beckmann et Jean-Henri Hassenfratz35. Un nouveau mode d'existence du phénomène technique émergeait. Nouveau parce que Diderot préconisait de prendre en compte le milieu gestuel dans sa totalité pour en extraire les rationalités et travailler en retour à les améliorer ce pourquoi il fallait la participation active et constante des «philosophes » (nous dirions aujourd'hui «scientifiques »). Nouveau parce que sa normativité débordait l'efficacité liée à la pratique située pour déterminer à partir d'elle, un principe d'efficacité applicable à d'autres ateliers, d'autres métiers et d'autres produits. L'efficacité devenait un concept.

D'une révolution industrielle à l'autre, le régime de la technologie trouve aujourd'hui son plein développement avec la conception industrielle assistée par ordinateur, qu'initia Pierre Bézier ${ }^{36}$. Le concepteur, non plus la machine ou l'ingénieur, est devenu le prescripteur. Un prescripteur dynamique, imaginant simultanément l'usage et le produit, adaptant la production au produit, et le process au geste technique.

«J'ai assisté au montage des planches de bord sur un véhicule », explique Pascal Daloz,
Directeur Général Adjoint de Dassault Systèmes chargé de la Stratégie et du Marketing, à propos de l'usage du logiciel CATIA chez Toyota ${ }^{37}$.

"L'équipe du soir, avant le repas, passait une demi-heure dans une salle remplie d'écrans pour apprendre avec des gants à retour de force à monter et démonter la planche de bord du futur modèle. Quand le geste n'était pas optimisé, un outil leur permettait d'expliquer leur problème et de proposer une modification de conception. La modification était modélisée et mise en œuvre dès le lendemain. De cette façon, le geste de l'ouvrier aiguille les concepteurs $» 38$.

C'est un double processus d'apprentissage qui est ici décrit : l'opérateur apprend les gestes que lui commandera ensuite la machine numérique pour la production d'un nouveau modèle; en retour, il apprend au développeur les insuffisances du programme numérique. "Ne pas tenir compte de ces éléments, condamne les concepteurs d'outils à rester en décalage permanent par rapport à l'activité industrielle. Il s'agit, au contraire, d'inscrire les outils dans l'action, ce qui implique aussi d'inscrire les possibilités d'action dans l'outil. Il importe donc de construire, à la fois, des outils donnant les moyens aux acteurs d'agir en mobilisant des connaissances anciennes et nouvelles et de rendre possible les nécessaires ajustements au sein de l'organisation » analyse Dominique Vinck 39 .

A l'opposé de l'OST qui détruit le milieu gestuel et réduit le travail au geste au nom de la productivité, le milieu gestuel devient la condition de la perfectibilité. On mesure toute l'ambiguïté de ce mode d'existence du geste technique : d'un côté, la programmation adapte la machine à l'homme, incorpore son savoir-faire; elle organise intimement l'interface homme-machine et peut l'individualiser s'il le faut; mais ce faisant, elle enferme le geste dans cet interface hybride. D'un côté, la programmation requiert l'expertise gestuelle qui ne saurait exister sans un milieu gestuel d'une grande richesse ${ }^{40}$. Mais, de l'autre, elle amenuise ce milieu gestuel en réduisant ses espaces conceptuels à des opérations virtuelles. Elle l'assujettit à cet 
usage que décide et impose le concepteur par la commande numérique ${ }^{41}$.

Les études anthropologiques menées autour de l'aviation de ligne, qui use abondamment du simulateur pour la formation de ses pilotes, éclaire sur les problèmes posés par la dématérialisation du geste technique. «On apprend pas à piloter en avion en simulateur », conclut Gérard Dubey d'une enquête conduite sur cette question. Et de citer un pilote de ligne : "Je pense qu'au simulateur les émotions sont artificielles... Quand on fait un beau tour de piste en avion, on a réussi quelque chose de concret $42[\ldots] »$ Parce qu'elle souligne spontanément, "naturellement » le lien entre esthétique, maîtrise de la pratique concrète et sentiment de réussite, cette courte observation est fondamentale. Tout geste technique s'inscrit dans un espace cognitif, dans une topique qui met en actes, en images, en sons et en mots, le réel sur lequel l'opérateur doit agir43. Mené à bien, il débouche sur une transformation matérielle, l'action sur la chose ou l'objet, et sur une sensation psychique, ce sentiment de satisfaction que décrit très bien le pilote de ligne («Quand on fait un beau tour de piste en avion, on a réussi quelque chose de concret [...]»). Une double mise en œuvre s'accomplit, celle de l'objet agi, parfaitement représenté par le chef d'œuvre des communautés de l'Ancien régime, et celle du sujet agissant, dont la technicité croissante, le fait passer d'apprenti à expert. Yves Schwartz dans ses travaux croisant philosophie et ergonomie a longuement insisté sur le caractère essentiel de l'accomplissement de soi, de «l'usage de soi » pour l'opérateur44. Richard Sennett a bien analysé l'importance du sentiment de «fierté » que provoquent le travail bien fait, et ses limites éthiques 45 .

C'est cette mise en œuvre que l'apprentissage des gestes techniques par simulateur ne parvient pas à restituer. Comme tout apprentissage, il met en jeu l'incorporation cognitive. Mais une incorporation tronquée. Le geste est là, mieux, il est suscité, requis, analysé, corrigé, répété jusqu'à l'automatisme. Mais son accomplissement n'a d'autre enjeu que l'apprentissage lui-même, et non l'action sur le réel. Moyens et fins sont dissociés. Le mode d'existence des gestes est comme inachevé : le virtuel n'engendre aucun régime de la pratique.

« Le simulateur est efficace pour tout ce qui se prête à répétition, tout exercice qu'il est possible de transposer dans une autre temporalité que celle du vol sans que cela affecte de façon notable son contenu. [...] Ce qui sanctionne la preuve irréfutable de son aptitude à piloter, c'est le réel, le caractère irréversible du temps réel », note Gérard Dubey46.

Or, il y a là une nécessité anthropologique.

« Autrement dit, observe Jean-Pierre Warnier, me dis-je moi qui travaille sur le rapport du corps à la culture matérielle : je constate qu'un pilote qui se situe dans la dernière période en date - l'humanité de la troisième révolution industrielle - reste néanmoins contraint par le fait qu'il est un sujet humain qui est un corps, un sujet incarné en tant que corps humain »47.

La réduction du milieu gestuel à la gestuelle opératoire, telle qu'elle se produit dans un régime de la technique développée à outrance dans le cadre industriel met l'opérateur en danger. La numérisation $\mathrm{du}$ geste, son intégration logicielle, telle qu'elle s'opère dans un régime de la technologie poussé dans ses retranchements logiques, économiques et commerciaux, nuit au renouvellement conceptuel des gestes ainsi désincarnés ${ }^{4}$. Ce régime opératoire se nourrit du geste expert en même temps qu'il crée les conditions de sa réification.

D’autres études sur les conditions de travail dans les centrales nucléaires ont mis à jour les effets négatifs de l'appauvrissement du geste technique sur l’identité-métier.

« Ce que je fais, il n'y a pas besoin d'un BTS pour le faire : tourner des vannes, faire des essais périodiques, suivre les gammes. Il existe des modes opératoires très stricts dans le nucléaire : ça ne laisse plus trop de place au professionnalisme des gens. » (jeune technicien, 30 ans).

Les jeunes, remarque Dominique Le Roux, auteur de l'enquête, n'ont plus guère l'occasion de pratiquer le geste technique dans la mesure où ce 
type d'activité prédomine. Seules les astreintes et les maintenances correctives leur permettent de pratiquer davantage et de " comprendre comment ça marche »49. Il y a là un facteur de risque à terme pour les transferts techniques et la transmission des savoir-faire informels que les spécialistes en Génie industriel connaissent bien. Les recherches se multiplient désormais, dans cette discipline, mais aussi en socio-anthropologie, en archéologie, en design, en gestion de l'innovation, pour contrecarrer le danger 50 .

$\mathrm{Au}$ bout de la route, sans doute y-a-t-il la mise en place d'un nouveau régime opératoire qui réintégrera la pratique et ses habitus au sein des environnements normatifs relevant de la technique et de la technologie. Ce renouvellement de l'efficacité sociotechnique, cette construction d'un nouveau mode d'existence du geste technique dans les sphères nouvelles du capitalisme cognitif, débordera vers le volet socioculturel, tant le geste est essentiel à la constitution identitaire ${ }^{51}$. L'histoire du geste technique participe de cette recherche quand elle analyse les attitudes, les matériaux, les outils et les chaines opératoires, quand elle fait l'étude comparative des milieux gestuels, quand elle détecte et observe l'historicité des modes d'existence que les régimes opératoires leur confèrent. Cela suppose de comprendre de manière plus dynamique que nostalgique, le triptyque histoire/mémoire/patrimoine.

${ }^{1}$ ROUX Valentine, BRIL Blandine, Stone Knapping: The Necessary Conditions for a Uniquely Hominin Behaviour, Cambridge, McDonald Institute for Archaeological Research, University of Cambridge, 2005.

${ }^{2}$ Charnière et non frontière. La découverte récente au Kenya d'un atelier de taille d'outils préhistoriques daté de 3,3 millions d'années et situé à proximité immédiate du site où a été découvert le Kenyanthrope Platyops conforte l'approche développée dans Stone Kapping. HARMAND Sonia et al., "3.3-millionyear-old stone tools from Lomekwi 3, West Turkana, Kenya »,
Nature,
mai
2015

(http://www2.cnrs.fr/presse/communique/4043.htm).
3 SIGAUT François, «Un couteau ne sert pas à couper, mais en coupant: structure, fonctionnement et fonction dans l'analyse des objets », 25 ans d'études technologiques en Préhistoire: bilan et perspectives; actes des rencontres, 18-19-20 oct. 1990, Centre de Recherches Archéologiques du CNRS, Antibes, Juanles-Pins, éditions APDCA, 1991, p. 21-34.

4 WARNIER Jean-Pierre, Construire la culture matérielle: l'homme qui pensait avec ses doigts, Paris, Presses universitaires de France, 1999, p. 125.

5 SIMONDON Michel, «L'ambiguïté de l'opération technique. Le geste technique et la vie », dans TINLAND Franck (dir.), Ordre biologique, ordre technologique, Seyssel, Champ Vallon, 1994, p. 102.

${ }^{6}$ BRIL Blandine et al., " Functional Mastery of Percussive Technology in Nut-Cracking and Stone-Flaking Actions: Experimental Comparison and Implications for the Evolution of the Human Brain », Philosophical Transactions of the Royal Society of London. Series B : Biological Sciences, vol. $367, \mathrm{n}^{\circ} 1585, \mathrm{p}$. 59-74.

7 "Les actes des XXIII ${ }^{e}$ Journées de Flaran rappellent donc opportunément que c'est dans le cadre de 'programmes de recherches coordonnées', dépassant les cadres nationaux et transcendant les périodisations universitaires, qu'une étape nouvelle pourra être franchie. Espérons que sera entendu le 'millionième appel à l'interdisciplinarité' lancé par John David au terme de sa présentation des sources de l'outillage médiéval » observe Patrick Fournier dans le compte-rendu qu'il fait en 2007 de l'ouvrage collectif sur l'outillage agricole médiéval et moderne et son histoire, édité par Georges Comet. FOURNIER Patrick, "Plaidoyer pour une histoire des gestes et de l'outil », Annales du Midi, t. 116, n⿳2026, 2004, p.233-236.

${ }^{8}$ GARÇON Anne-Françoise, « Du danger de l'archive écrite en histoire des techniques ", e-Phaïstos, vol.2, $\mathrm{n}^{\circ} 2,2013$, p.10-27.

9 Projet Bretez : https://sites.google.com/site/louisbretez/home. Dernière version disponible de la maquette sonore : 30/05/2014) : http://youtu.be/YP_1eHeyo4.

10 CAUUET Béatrice, « Gold and silver extraction in Alburnus Maior mines, Roman Dacia (Rosia Montana, Romania). Dynamics of exploitation and management of the mining space ", dans FONTES L. et MARTINS C. M. B. (dir.), Paisagens mineiras antigas na Europa occidental. Investigaçao e Valorizaçao Cultural, Boticas, 2014, p. 82-104; ARLES Adrien et al., «3D Reconstruction and Modeling of Subterranean Landscapes in Collaborative Mining Archaeology Projects : Techniques, Applications and Experiences ", International Archives of the Photogrammetry, Remote Sensing and Spatial Information Sciences, vol. XL, n ${ }^{\circ} 5 / \mathrm{W} 2,2013$, p. 61-66.

${ }^{11}$ PERNOT Michel, «Techniques, ateliers et artisans du 'bronze' dans l'Aquitaine antique de la fin de l'âge du Fer et de la période 
gallo-romaine », ADLFI. Archéologie de la France-Informations, Aquitaine, mis en ligne le 01 mars 2008, http://adlfi.revues.org/2681.

${ }_{12}$ MICHEL Alain P., « La reconstitution virtuelle d'un atelier de Renault-Billancourt : sources, méthodologie et perspectives ", Documents pour l'histoire des techniques $\mathrm{n}^{\circ} 18$, 2e semestre 2009, p. 23-38.

${ }^{13}$ CAILLOCE Laure, " La Grotte Chauvet vue par ses copistes », CNRS. Le Journal, 24 avril 2015, https://lejournal.cnrs.fr/articles/la-grotte-chauvet-vue-par-sescopistes.

${ }^{14}$ Utile précision : mon travail sur G. Agricola s'est fait en croisant le texte original en latin traduit par mes soins et la traduction anglaise de référence signée d'Herbert Hoover.

${ }_{15}$ Sur les conclusions scientifiques de cette absence, voir mes articles « Pratique, technique, technologie ? ", ArchéoSciences, $\mathrm{n}^{\mathrm{o}} 34,2010$, p. 121-126 et « Réduire la mine en science ? Anatomie des De re metallica d'Agricola (1528-1556) », dans DUBOURG-GLATIGNY P. et VÉRIN H. (dir.), Réduire en ar, p. 317336.

16 BRIOIST Pascal, "La réduction en art de l'escrime au XVI siècle », Ibidem, p.293-316.

${ }_{17}$ ROBERT Luis, Le judo et la self-défense Des méthodes de défense... aux techniques de combat..., Marabout Flash $\mathrm{n}^{\circ} 94$, Verviers, Editions Gérard et $C^{\circ}, 1962$; ROLAND Ghislaine et Georges, Tennis de table. Du Ping-pong à la compétition, Collection Marabout Flash n ${ }^{\circ} 84$, Verviers, Editions Gérard et C $C^{\circ}, 1961$.

18 « Le régime opératoire, qui est la technicité en acte, a son sens normatif en amont de l'application d'un calcul, à partir des seuils de fonctionnement reliant l'ordre technologique à son mode associé, aux potentiels des formes du champ opératoire qui n'est pas analogues à un espace homogène et isomorphe, aux exigences aussi de la vie en ses gestes. On pourrait viser ainsi à faire l'archéologie, la genèse et la phénoménologie des régimes techniques d'activité... », SIMONDON Michel, «L'ambiguïté... », in TINLAND F. (dir.), Ordre... op. cit., p. 105.

${ }^{19}$ La question posée ici est celle de la « tendance technique », qui relève de l'histoire technique des techniques.

${ }^{20}$ La question posée ici est celle de l'efficacité, qui relève de l'histoire sociale et culturelle des techniques.

${ }^{21}$ Sur la voile et l'art du barreur, voir les beaux développements de WARNIER J.-P. dans l'introduction de Construire la culture matérielle..., op.cit., p. 10-13.

${ }^{22}$ DUBOURG-GLATIGNY Pascal Dubourg-Glatigny et VÉRIN Hélène, Réduire..., op. cit., passim.

23 Le dévoilement intervint en France entre les années 1820 et les années 1840 , sous la plume des élèves-ingénieurs des mines qui ne se retiraient pas au moment de la finition comme les aides avaient coutume de le faire, et consignaient la totalité du procédé et des gestes du maître-coupelleur dans leurs Mémoires et Journaux de voyage. GARÇON A.-F., «Un essai d'anthropologie technique : l'art du coupelleur ", dans Les métaux non ferreux en France aux XVIII et XIXe siècles. Ruptures, blocages, évolutions au sein des systèmes techniques, Thèse EHESS, dactyl., 1995, vol. 1, p. 202-208. En ligne, https://halshs.archivesouvertes.fr/halshs-00008537.

24 Ce qu'explique très bien Hélène Vérin : «L'émergence du domaine de l'art, c'est-à-dire de procédures de validation des choix techniques appuyées sur des raisons à caractère démonstratif, tend à modifier les modes de négociation et de coopération entre les différents protagonistes concernés. En particulier dans les arts mécaniques, c'est une nouvelle distribution des compétences et des responsabilités qui s'instaure entre commanditaires, experts et hommes de métier, surtout lorsqu'il s'agit de projets d'envergure », VÉRIN Hélène, " Rédiger et réduire en art : un projet de rationalisation des pratiques ", dans DUBOURGGLATIGNY P. et VÉRIN H., Réduire... op. cit., p. 52.

25 BRIOIST Pascal, DRÉVILLON Hervé, SERNA Pierre, Croiser le fer: violence et culture de l'épée dans la France moderne (XVI ${ }^{e}-X V I I I^{e}$ siècle), Seyssel, Champ Vallon, 2002.

26 « L'un des lieux de cette confrontation entre experts et métiers est le travail sur les mots et les définitions dans les réductions en art », VÉRIN Hélène, « Rédiger... » op. cit..

${ }_{27}$ VÉRIN Hélène, « Spectacle ou expérimentation : La préfabrication de vaisseaux en 1679 », Revue de Synthèse, vol. 108, $\mathrm{n}^{\circ}$ 2, 1987, p. 199-223.

28 DELAUNAY Bernard, La technique à l'Académie royale des sciences (1699-1750), Paris, Éditions H. Champion, 2016.

29 PEAUCELLE Jean-Louis, « La division du travail. Adam Smith et les encyclopédistes observant la fabrication des épingles en Normandie », Gérer et Comprendre. Annales des Mines, Septembre 1999, p. $35-51$; « Les innovations techniques et organisationnelles dans la fabrication des épingles », Innovations vol. 1, $\mathrm{n}^{\circ} 27,2008$, p. 27-44.

${ }^{30} \mathrm{Il}$ est difficile à ce stade de savoir s'il s'agit d'une caractéristique essentielle, structurelle, propre au régime de la technique, toutes époques et cultures confondues, ou d'une caractéristique située, propre à la forme qu'en a donné la culture européenne.

${ }^{31}$ GARÇON Anne-Françoise, « Du travail comme non-valeur philosophique ", dans L'Imaginaire et la pensée technique. Une approche historique, $X V I^{e}-X X^{e}$ siècle, Paris, Classiques Garnier, 2012, p. 101-113.

${ }^{32}$ Gottfried Wilhelm Leibniz, Protogaea. Translation and Introduction by Claudine Cohen and Andre Wakefield, Chicago, University of Chicago Press, 2008. 
33 GARÇON Anne-Françoise, "The Three States of Technology: An Historical Approach to a Thought Regime, $16^{\text {th }}-20^{\text {th }}$ Centuries ", dans FAUCHEUX M. et FOREST J. (eds), New Elements of Technology, Belfort-Montbéliard, Presses de l'UTBM, 2012, p. 11-26.

34 «L'histoire des Arts que nous avons entrepris d'écrire n'est point celle de leur progrès, de leur décadence, mais celle des pratiques qui sont actuellement en usage; nous avons appelé cette espèce de description 'histoire des Arts', comme on nomme 'histoire naturelle' la description des productions de la nature », écrivait Réaumur dans une préface des Descriptions demeurée inédite. Cité par JAOUL Martine et PINAULT-SØRENSEN Madeleine, «La collection 'Description des Arts et Métiers' Étude des sources inédites de la Houghton Library Université Harvard », Ethnologie française, t. 12, $n^{\circ}$ 4, 1982, p. 353.

35 GUILLERME Jacques et SEBESTIK Jan, «Les commencements de la technologie », Thalès, t. 12, année 1966, p. 1-72, reprint : Documents pour l'Histoire des Techniques, $\mathrm{n}^{\circ}{ }^{14}, 2007$, p. 49-121; LABOULAIS Isabelle, La Maison des Mines : la genèse révolutionnaire d'un corps d'ingénieurs civils, 1794-1814, Rennes, Presses universitaires de Rennes, 2012.

${ }^{36}$ Les actes du colloque international Pierre Bézier et les machines-outils (\& machine tools), 15-17 septembre 2010, Evry, Paris, France, sont sous presse (Alain P. Michel éd.).

37 DALOZ Pascal et al., « Concevoir les outils du bureau d'études : Dassault Systèmes, Une firme innovante au service des concepteurs », Entreprises et histoire, ${ }^{\circ} 58,2010$, p.150-164. Le logiciel CATIA de conception assistée tridimensionnelle interactive est l'une des grandes réussites de Dassault Systèmes.

${ }^{38}$ Ibidem.

39 VINCK Dominique, « Ethnographie des activités de conception et d'innovation : le cas du maquettage numérique », Management technologique. Impact de la technologie sur la gestion des personnes, $12^{\mathrm{e}}$ Entretiens Jacques Cartier, Décembre 1999, Grenoble, France, Actes disponibles uniquement sur CD-ROM, 1999. En ligne : https://hal.archives-ouvertes.fr/hal-o0134429.

40 Nous sommes très exactement dans la "coprescription » nécessaire au processus de conception, décrite par HATCHUEL Armand dans "Apprentissages collectifs et activité de conception ", Revue Française de Gestion, juillet 1994, p.109-120 et analysée par D. Vinck.

${ }^{41}$ Il en va de même du geste de l'usager, conduit, pour ne pas dire contraint, par le design de l'objet.

42 DUBEY Gérard, «La simulation informatique à l'épreuve de l'altérité », Socioanthropologie, $\mathrm{n}^{\circ} 3,1998$, p.49-65 ; « La simulation à l'épreuve du lien social », Le travail humain, vol. $64, \mathrm{n}^{\circ} 1$, 2001, p.3-28; GRAS Alain, MORICOT Caroline, POIROTDELPECH Sophie et SCARDIGLI Victor, Face à l'automate : le pilote, le contrôleur et l'ingénieur, Paris, Publications de la
Sorbonne, 1994. Il est intéressant de noter que, de son propre aveu, le commandant de bord qui évita le pire en faisant amerrir son avion dans la rivière Hudson, a pu accomplir ce tour de force technique grâce à sa connaissance approfondie du vol à voile.

43 C'est dans cette topique que se forge la capacité à anticiper. Ce qui fait qu'en définitive, un marteau sert en frappant certes, mais aussi, à frapper de toutes les manières parce que la technicité acquise et/ou transmise, rend possible l'invention.

44 SCHWARTZ Yves, Travail et philosophie. Convocations mutuelles, Toulouse, Octarès, 1992, p. 45-66. - Cela rejoint l'eupraxie, le «bien faire » qui caractérise la technologie telle qu'elle était enseignée aux Etats-Unis au XIXe siècle.

45 SENNETT Richard, Ce que sait la main. La culture de l'artisanat, Paris, A. Michel, 2010, p. 394-396.

46 DUBEY Gérard, "La simulation informatique... », Socioanthropologie, op.cit., p.50-51. - «On ne peut sans dommage annuler l'historique dans le travail », confirme SCHWARTZ Yves, Travail..., op.cit., p. 75 .

47 WARNIER Jean-Pierre, «Diane chasseresse. Entretien avec Jean-Luc Evrard », Flavius : la Quinzaine Géopolitique, en ligne, http://www.flavius.eu/.

${ }^{48} \mathrm{Au}$ sortir de son analyse ethnographique du maquettage numérique dans les bureaux d'étude, D. Vinck préconisait, «le développement d'espace d'apprentissage au sein desquels les acteurs (à la fois sur l'axe « concepteurs - utilisateurs » des outils et sur l'axe des métiers intervenant dans la conception), de manière précoce, puissent faire connaître leurs contraintes. Nous sommes donc tout à l'opposé d'une logique de rationalisation et d'optimisation de l'activité de conception, à coup d'outils de maquettage numérique, de programmes de formation pour adapter les individus aux outils et de programmes de réorganisation pensés à partir des outils. Au contraire, tant au niveau de la conception des outils de conception qu'au niveau de leur usage dans la conception de produits, il s'agit de permettre aux acteurs d'inventer les outils qui facilitent la construction des compromis entre les exigences contradictoires dont ils sont les porte-parole et qui rendent également possible la mémorisation des contraintes de chacun et celle des compromis construits ». VINCK D., «Ethnographie... », op.cit.

49 LE ROUX Dominique, « Les processus sociaux de la transmission intergénérationnelle des compétences : le cas d'une centrale nucléaire ", Sociologies pratiques, vol. 1, $\mathrm{n}^{\circ} 12$, 2006, p. 23-36.

${ }^{50}$ Le recours aux «serious games » est l'un des moyens actuellement développés pour dépasser la difficulté.

${ }^{51}$ Le rôle du bricolage, du sport, de la pêche, du jardinage, n'est pas seulement économique ou culturel. 